\title{
Comparison theorems between algebraic and analytic De Rham cohomology (with emphasis on the $p$-adic case)
}

\author{
par YVES ANDRÉ
}

\begin{abstract}
RÉSUMÉ. Nous présentons un panorama des théorèmes de comparaison entre les cohomologies de De Rham algébrique et analytique à coefficients dans des connections algébriques. Ces théorèmes ont joué un rôle important dans le développement de la théorie des $\mathcal{D}$-modules, en particulier dans l'étude de leurs propriétés de ramification (irrégularité...). Dans la partie I, nous nous concentrons sur le cas des coefficients réguliers et esquissons la nouvelle preuve de ces théorèmes donnée par F. Baldassarri et l'auteur, qui est de nature élémentaire et unifie les théories complexe et $p$-adique. Dans le cas $p$-adique cependant, le théorème de comparaison était supposé s'étendre aux coefficients irréguliers et ceci a été prouvé dans $[\mathrm{AB}]$. La preuve de cette extension suit le même modèle que pour le cas régulier, mais demande en supplément une étude détaillée de l'irrégularité en plusieurs variables. Dans la partie II, nous donnons un aperçu de cette preuve qui peut servir de guide pour le livre $[A B]$.
\end{abstract}

ABSTRACT. We present a panorama of comparison theorems between algebraic and analytic De Rham cohomology with algebraic connections as coefficients. These theorems have played an important role in the development of $\mathcal{D}$-module theory, in particular in the study of their ramification properties (irregularity...). In part I, we concentrate on the case of regular coefficients and sketch the new proof of these theorems given by F. Baldassarri and the author, which is of elementary nature and unifies the complex and $p$-adic theories. In the $p$-adic case, however, the comparison theorem was expected to extend to irregular coefficients, and this has recently been proved in $[\mathrm{AB}]$. The proof of this extension follows the same pattern as in the regular case, but involves in addition a detailed study of irregularity in several variables. In part II, we give an overview of this proof which can serve as a guide to the book $[\mathrm{AB}]{ }^{1}$

\footnotetext{
1 added on proofs: a second (revised) edition of $[A B]$ is in preparation.
} 


\section{A new approach to the Grothendieck-Deligne comparison theorems for De Rham cohomology (and its $p$-adic counterpart)}

\subsection{Introduction.}

The idea of computing the cohomology of a manifold, in particular its Betti numbers, by means of differential forms goes back to E. Cartan and G. De Rham. In the case of an algebraic manifold, there is the possibility of using only algebraic differential forms, and the problem arises whether this gives rise to the right cohomology groups. I. Petrovsky had already encountered this problem in his studies of real algebraic geometry, and it had been considered (and solved) independently by G. Hochschild and B. Kostant in the case of affine homogeneous manifolds; the problem has been solved in the general case in 1963 by A. Grothendieck.

The most concrete instance of Grothendieck's theorem reads as follows: let $Y$ be a closed differentiable submanifold of $\mathbf{C}^{n}$ defined by polynomial equations

$$
P_{1}\left(T_{1}, \ldots, T_{n}\right)=\ldots=P_{s}\left(T_{1}, \ldots, T_{n}\right), \quad P_{j} \in \mathbf{C}\left[T_{1}, \ldots, T_{n}\right] .
$$

An algebraic differential form $\alpha$ on $Y$ of degree $p$ is the restriction to $Y$ of a rational differential form of degree $p$ in the variables $T_{1}, \ldots, T_{n}$, which has no pole on $Y$. It is closed if $d \alpha=0$ on $Y$.

Theorem (Grothendieck). Any closed $C^{\infty}$ differential form on $Y$ is the sum $\alpha+d \eta$ of a closed algebraic differential form $\alpha$ and an exact $C^{\infty}$ differential form $d \eta$. Moreover, If $d \eta$ is algebraic, then $d \eta=d \beta$ for some algebraic differential form $\beta$.

Thus transcendental and algebraic De Rham cohomology groups coincide, and as a consequence, the Betti numbers of $Y$ can be computed purely algebraically. Grothendieck's proof uses Hironaka's resolution of singularities in an essential way [G].

In order to discuss this result and some generalization in more detail, let us introduce some notation.

Let $X$ be a smooth complex algebraic variety of dimension $d$. Let $\Omega_{X}^{p}=$ $\Lambda^{p} \Omega_{X}^{p}$ be the sheaf of algebraic differential $p$-forms on $X$. These sheaves fit into the (augmented) De Rham complex

$$
(\mathbf{C} \rightarrow) \Omega_{X}^{0} \rightarrow \Omega_{X}^{1} \rightarrow \Omega_{X}^{2} \rightarrow \ldots
$$

Similarly, one can build the (augmented) De Rham complex attached to the analytic manifold $X^{a n}$ underlying $X$

$$
(\mathrm{C} \rightarrow) \Omega_{X^{a n}}^{0} \rightarrow \Omega_{X^{a n}}^{1} \rightarrow \Omega_{X^{a n}}^{2} \rightarrow \ldots
$$


and the (augmented) De Rham complex attached to the differentiable manifold $X^{\text {an }}$ underlying $X$

$$
(\mathbf{C} \rightarrow) \Omega_{X^{\infty}}^{0} \rightarrow \Omega_{X^{\infty}}^{1} \rightarrow \Omega_{X^{\infty}}^{2} \rightarrow \ldots
$$

Note that the first two complexes stop at $\Omega^{d}$ (i.e. $\Omega_{X}^{d+1}=\Omega_{X^{a n}}^{d+1}=0$ ) while the third one stops only at $\Omega^{2 d}$. On the other hand, the last two augmented complexes (but not the first one) are exact by the Poincaré lemma.

According to the standard definition, the De Rham cohomology of $X^{\infty}$ is the cohomology of the complex of global sections

$$
\Gamma \Omega_{X^{\infty}}^{0} \rightarrow \Gamma \Omega_{X^{\infty}}^{1} \rightarrow \Gamma \Omega_{X^{\infty}}^{2} \rightarrow \ldots
$$

However, because the $\Omega_{X^{\infty}}^{p}$ are fine sheaves, this is the same as the hypercohomology of the $C^{\infty}$ De Rham complex

$$
H_{d R}^{*}\left(X^{\infty}\right)=\mathbf{H}^{*}\left(\Omega_{X^{\infty}}^{0} \rightarrow \Omega_{X^{\infty}}^{1} \rightarrow \Omega_{X^{\infty}}^{2} \rightarrow \ldots\right)
$$

In the analytic and algebraic cases, one defines De Rham cohomology by

$$
H_{d R}^{*}\left(X^{a n}\right)=\mathbf{H}^{*}\left(\Omega_{X^{a n}}^{0} \rightarrow \Omega_{X^{a n}}^{1} \rightarrow \ldots\right), H_{d R}^{*}(X)=\mathbf{H}^{*}\left(\Omega_{X}^{0} \rightarrow \Omega_{X}^{1} \rightarrow \ldots\right) \text {. }
$$

These various De Rham cohomology groups are related by natural maps

$$
H_{d R}^{*}(X) \longrightarrow H_{d R}^{*}\left(X^{a n}\right) \longrightarrow H_{d R}^{*}\left(X^{\infty}\right) .
$$

Due to the exactness of the analytic and $C^{\infty}$ augmented De Rham complexes, the last map is an isomorphism and in fact

$$
H_{d R}^{*}\left(X^{a n}\right) \cong H^{*}(X(\mathbf{C}), \mathbf{C}) \cong H_{d R}^{*}\left(X^{\infty}\right) .
$$

Now, Grothendieck's comparison theorem tells us that the first map $H_{d R}^{*}(X) \longrightarrow H_{d R}^{*}\left(X^{a n}\right)$ is also an isomorphism.

Grothendieck has also raised the problem of generalizing this comparison theorem in the case of non-constant coefficients. Let $\mathcal{M}$ be an algebraic vector bundle on $X$ endowed with an integrable connection

$$
\nabla: \mathcal{M} \longrightarrow \Omega_{X}^{1} \otimes \mathcal{M}
$$

(a C-linear morphism of abelian sheaves satisfying Leibniz' rule, and such that the map $D \in \mathcal{T}_{X}=\left(\Omega_{X}^{1}\right)^{\vee} \mapsto \nabla_{D}=(D \otimes i d) \circ \nabla \in E n d_{\mathbf{C}-l i n} \mathcal{M}$ commutes with the brackets on $\mathcal{T}_{X}$ and $\left.E n d_{\mathbf{C}-\text { lin }} \mathcal{M}\right)$.

By means of the connection, one constructs in the standard way a complex with C-linear differential (the De Rham complex of $(\mathcal{M}, \nabla)$ :

$$
\mathcal{M} \rightarrow \Omega_{X}^{1} \otimes \mathcal{M} \rightarrow \Omega_{X}^{2} \otimes \mathcal{M} \rightarrow \ldots
$$

The De Rham cohomology of $X$ with coefficients in $(\mathcal{M}, \nabla)$ is defined to be the hypercohomology of this complex.

Similarly, one can build the De Rham complex attached to the analytic manifold $X^{a n}$ underlying $X$

$$
\mathcal{M}^{a n} \rightarrow \Omega_{X^{a n}}^{1} \otimes \mathcal{M}^{a n} \rightarrow \Omega_{X^{a n}}^{2} \otimes \mathcal{M}^{a n} \rightarrow \ldots
$$


and there is a natural map of hypercohomologies

$$
H_{d R}^{*}(X,(\mathcal{M}, \nabla)) \longrightarrow H_{d R}^{*}\left(X^{a n},\left(\mathcal{M}^{a n}, \nabla^{a n}\right)\right) .
$$

This map is not an isomorphism in general, even in degree 0: indeed, let for instance $X$ be the affine line with coordinate $x, \mathcal{M}=\mathcal{O}_{X}$, and let $\nabla$ be the connection given by $\nabla(1)=d x$; then

$$
\begin{aligned}
H_{d R}^{0}(X,(\mathcal{M}, \nabla)) & =\operatorname{Ker} \nabla=0 \\
H_{d R}^{0}\left(X^{a n},\left(\mathcal{M}^{a n}, \nabla^{a n}\right)\right) & =\operatorname{Ker} \nabla^{a n}=\text { C. } e^{-x} .
\end{aligned}
$$

In this counter-example, the connection has only one singularity at infinity, which is irregular, i.e. not regular (cf. [M]). For De Rham cohomology with regular coefficients, P. Deligne has proved the following generalization of Grothendieck's comparison theorem:

Theorem (Deligne). Assume that $\nabla$ is regular (at every divisor at infinity). Then the map $H_{d R}^{*}(X,(\mathcal{M}, \nabla)) \rightarrow H_{d R}^{*}\left(X^{a n},\left(\mathcal{M}^{a n}, \nabla^{a n}\right)\right)$ is an isomorphism.

Deligne's strategy of proof goes as follows [D]:

i) construct a Hironaka compactification of $X: \bar{X}$ proper and smooth, $\bar{X} \backslash X=$ divisor with normal crossings;

ii) extend $\mathcal{M}$ to a vector bundle $\overline{\mathcal{M}}$ on $\bar{X}$ and $\nabla$ to a connection $\bar{\nabla}$ with logarithmic poles along $\bar{X} \backslash X$ (using the regularity assumption);

iii) by GAGA, the natural map

$$
H_{d R, \log }^{*}(\bar{X},(\overline{\mathcal{M}}, \bar{\nabla})) \rightarrow H_{d R, l o g}^{*}\left(\bar{X}^{a n},\left(\overline{\mathcal{M}}^{a n}, \bar{\nabla}^{a n}\right)\right)
$$

is an isomorphism;

$i v$ ) by studying ramification, compare

$$
H_{d R}^{*}(X,(\mathcal{M}, \nabla)) \text { and } H_{d R, l o g}^{*}(\bar{X},(\overline{\mathcal{M}}, \bar{\nabla}))
$$

$\left(\right.$ resp. $H_{d R}^{*}\left(X^{a n},\left(\mathcal{M}^{a n}, \nabla^{a n}\right)\right)$ and $\left.H_{d R, l o g}^{*}\left(\bar{X}^{a n},\left(\overline{\mathcal{M}}^{a n}, \bar{\nabla}^{a n}\right)\right)\right)$.

Point $i v)$, comparison between a meromorphic De Rham complex and a De Rham complex with essential singularities at infinity, is the crux of the proof. The difference between those two De Rham complexes has been analysed in a systematic way by Z. Mebkhout; in his geometric theory of irregularity, it is measured by a certain perverse sheaf [Me1]. This has eventually led him to a new proof [Me2] of the Grothendieck-Deligne theorem which bypasses point $i$ ); in other words, it is no longer necessary to invoke resolution of singularities, as a deus ex machina, to investigate any serious question of ramification arising in the theory of $\mathcal{D}$-modules.

Non-archimedean analogues of the Grothendieck and Deligne comparison theorems have been proved by R. Kiehl [Ki] and F. Baldassarri [B3] 
respectively. In this context, $X$ is smooth algebraic variety over $\mathbf{C}_{p}=\hat{\overline{\mathbf{Q}}}_{\mathbf{p}} \cdot{ }^{2}$

Recently, F. Baldassarri and the present author have looked for a common proof of the complex and $p$-adic comparison theorems. Of course, such a proof has to avoid the (typically transcendental complex) notion of monodromy. This has led us to a new, elementary, and more algebraic treatment of the Grothendieck-Deligne theorem (which does not use resolution of singularities) $[\mathrm{AB}]$. We present here a sketch of this proof.

\subsection{Relative De Rham cohomology and dévissage.}

1.2.1. We first need a relative version of De Rham cohomology (higher direct images). Let $f: X \rightarrow S$ be a smooth morphism of smooth complex algebraic varieties, of pure relative dimension $d$ ( $S$ being connected for convenience).

To any $\mathcal{O}_{X}$-module with integrable connection $(\mathcal{M}, \nabla)$, one attaches the relative De Rham complex $\Omega^{*}(\mathcal{M})$ (usually, $\mathcal{M}$ will be a vector bundle, so that $\Omega^{*}(\mathcal{M})$ is a complex of vector bundles with $f^{-1}\left(\mathcal{O}_{S}\right)$-linear differential).

This complex only involves the relative connection $\nabla_{\text {rel }}: \mathcal{M} \rightarrow \Omega_{X / S}^{1} \otimes \mathcal{M}$ induced by $\nabla$; the fact that $\nabla_{\text {rel }}$ arises from an absolute integrable connection $\nabla$ is reflected by the existence of a canonical integrable connection (the Gauss-Manin connection $[\mathrm{KO}]$ ) on the $\mathcal{O}_{S}$-modules of hypercohomology

$$
R_{d R}^{i} f_{*}(\mathcal{M}, \nabla):=\mathbf{R}^{i} f_{*}\left(\Omega^{*}(\mathcal{M})\right) .
$$

It has been recently checked that this construction agrees, up to a cohomological shift by $d$, with the construction of smooth direct images in $\mathcal{D}$-module theory [DMSS]1.4.

Examples. $R_{d R}^{0} f_{*}(\mathcal{M}, \nabla)=\operatorname{Kerf}_{*} \nabla_{\text {rel }}$. If $f$ is affine and $d=1$, $R_{d R}^{1} f_{*}(\mathcal{M}, \nabla)=\operatorname{Cokerf}_{*} \nabla_{\text {rel }}$ and $R_{d R}^{i} f_{*}(\mathcal{M}, \nabla)=0$ for $i \neq 0,1$.

1.2.2. Our strategy of proof of the comparison theorem in De Rham cohomology is reminiscent of M. Artin's proof of the comparison theorem in étale cohomology $[\mathrm{Ar}]$, using dévissage and reduction to the case of socalled "elementary fibrations". Instead of compactifying as in Deligne's proof, we "localize" $X$ to the effect that the fibers of the morphism $X \rightarrow S$ "become $K(\pi, 1)$ 's".

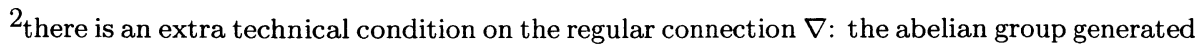
by 1 and the exponents at infinity should contain no Liouville number, a condition which is automatically satisfied if for instance $X$ and $(\mathcal{M}, \nabla)$ are defined over $\overline{\mathbf{Q}}$.
} 
Definition (Artin). $f$ is an elementary fibration if it can be embedded in a commutative diagram

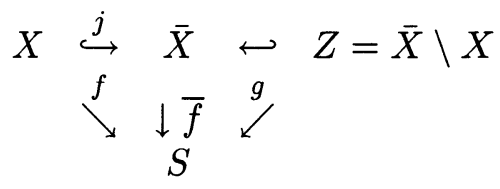

where

(i) $Z$ is a closed reduced subscheme of $\bar{X}$ and $j$ is the complementary open immersion;

(ii) $\bar{f}$ is projective and smooth with geometric fibers irreducible of dimension 1 ;

(iii) $g$ is an étale covering.

Definition. An elementary fibration $f$ is called rational if $\bar{X}=\mathbf{P}^{1} \times S, \bar{f}=$ $p r_{2}$, and $Z$ is a disjoint union of images of sections $\sigma_{i}, i=1, \ldots, r, \infty\left(s_{\infty}\right.$ : $S \rightarrow Z_{\infty}=\{\infty\} \times S$ ).

Remarks. An elementary fibration is a surjective affine morphism. Rational elementary fibrations are relative versions of "the affine line minus a few points".

Proposition 1.2.3 (Artin). Let $f: X \rightarrow S$ be a smooth morphism as before. There is an étale dominant morphism $\epsilon: S^{\prime} \rightarrow S$ and a finite open cover $\left(U_{\alpha}\right)$ of $X \times_{S} S^{\prime}$ such that each $U_{\alpha} \rightarrow S^{\prime}$ is a tower of elementary fibrations.

We now consider certain properties $\mathcal{P}$ of modules with integrable connection, which satisfy the following conditions:

- $\mathcal{P}$ is local for the étale topology,

- $\mathcal{P}$ is exact: if $0 \rightarrow\left(\mathcal{M}_{1}, \nabla_{1}\right) \rightarrow(\mathcal{M}, \nabla) \rightarrow\left(\mathcal{M}_{2}, \nabla_{2}\right) \rightarrow 0$ is exact, $(\mathcal{M}, \nabla)$ satisfies $\mathcal{P}$ if $\left(\mathcal{M}_{1}, \nabla_{1}\right)$ and $\left(\mathcal{M}_{2}, \nabla_{2}\right)$ do,

- $\mathcal{P}$ implies coherence of the underlying module.

We are interested in the stability of $\mathcal{P}$ under direct image $R_{d R}^{i} f_{*}$.

Lemma (dévissage). Assume that for any rational elementary fibration $f: X \longrightarrow S$ with affine base, and for any $\mathcal{O}_{X}$-module with integrable connection $(\mathcal{M}, \nabla)$ satisfying $\mathcal{P}$, there is a dense open subset $U \subset S$ such that $R_{d R}^{i} f_{*}(\mathcal{M}, \nabla)_{\mid U}$ also satisfies $\mathcal{P}$ in degree $i=0,1$.

Then the same is true for any smooth morphism $f$ in any degree $i$.

Steps of the proof ([AB]III.3.3): $i$ ) Cech spectral sequence for De Rham cohomology with respect to the cover $\left(U_{\alpha}\right)$ of $X \times{ }_{S} S^{\prime}$; 
ii) The Leray spectral sequence for the tower $U_{\alpha} \rightarrow S^{\prime}$ reduces the question to the case when $f$ is an elementary fibration (in which case $R_{d R}^{i} f_{*}(\mathcal{M}, \nabla)=$ 0 for $i \neq 0,1) ;^{3}$

iii) Applying suitable finite mappings (using Lefschetz' pencils), one further reduces to the case when $f$ is a rational elementary fibration.

Variants: • the assumption of the lemma can be weakened by restricting to "cyclic" connections with respect to a vertical coordinate;

- if one can take $U=S$ in the assumption, it is then possible to take $U$ independent of $(\mathcal{M}, \nabla)$ (but not $=S$ ) in the conclusion.

\subsection{On the coherence of direct images.}

As an illustration of the previous lemma, let us take $\mathcal{P}=$ coherence of the underlying $\mathcal{O}$-module. For $\mathcal{O}$-modules with integrable connection, it is well-known that coherence is equivalent to being a vector bundle (cf. [K1]).

Theorem 1.3.1. Let $f: X \rightarrow S$ be a smooth morphism, and $(\mathcal{M}, \nabla)$ be a vector bundle with integrable connection. Then there is an open dense subset $U \subset S$ such that $R_{d R}^{i} f_{*}(\mathcal{M}, \nabla)_{\mid U}$ is a vector bundle for every $i$.

The usual proof of this result uses the notion of holonomy and its stability under direct image (cf. $[\mathrm{Bn}][\mathrm{Bo}])$.

1.3.2. Let us sketch a proof along the previous dévissage (with $\mathcal{P}=$ coherence) cf. [AB]III.4.1. It suffices to prove the theorem when $f$ is a rational elementary fibration with affine $S, \nabla$ is cyclic (with respect to a vertical coordinate), and $i=0,1$

$$
\begin{array}{cccc}
X & \stackrel{j}{\hookrightarrow} & \mathbf{A}_{S}^{1} & \hookleftarrow
\end{array} \quad Z=\coprod_{i}\left(x=\theta_{i}\right)
$$

with $\theta_{i}-\theta_{j} \in \mathcal{O}(S)^{*}, x$ being the "vertical" coordinate on $\mathbf{A}^{1}$. Decomposition of rational functions into principal parts yields

$$
\mathcal{O}(X)=\mathcal{O}(S)[x] \oplus \bigoplus_{i} \frac{1}{x-\theta_{i}} \mathcal{O}(S)\left[\frac{1}{x-\theta_{i}}\right]
$$

Our connection $\nabla$ is given by a differential operator

$$
\Lambda=\sum_{0}^{\mu} \gamma_{k} \frac{d^{k}}{d x^{k}} \in \mathcal{O}(X)\left[\frac{d}{d x}\right]
$$

and we may assume that $\gamma_{\mu} \in \mathcal{O}(X)^{*} \cap \mathcal{O}(S)[x]$. We have

$$
R_{d R}^{0} f_{*}(\mathcal{M}, \nabla)=\operatorname{Ker} \Lambda, R_{d R}^{1} f_{*}(\mathcal{M}, \nabla)=\operatorname{Coker} \Lambda
$$

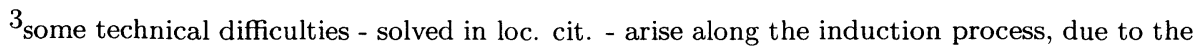
fact that $\mathcal{P}$ is only assumed generically.
} 
and we have to show that these $\mathcal{O}(S)$-modules are finitely generated. The case of $\operatorname{Ker} \Lambda$ being easy, we concentrate on $\operatorname{Coker} \Lambda$.

1.3.3. In order to analyse $\operatorname{Coker} \Lambda$, we need the notion of indicial polynomial.

Definition. The indicial polynomial of $\Lambda$ at $\theta_{i}$ is the unique polynomial $\phi_{i} \in \mathcal{O}(S)[t]$ in the variable $t$ with the following property: there is an integer $r_{i}$ such that for every $m$,

$$
\Lambda \frac{1}{\left(x-\theta_{i}\right)^{m}}=\phi_{i}(-m) \frac{1}{\left(x-\theta_{i}\right)^{m+r_{i}}}+\text { lower order terms in } \frac{1}{x-\theta_{i}} .
$$

The roots of the indicial polynomial are called the (Fuchs) exponents of $\Lambda$ at $\theta_{i}$.

Lemma (Robba). Due to the integrability of $\nabla$, the exponents of $\Lambda$ at $\theta_{i}$ are constant.

cf. $[\mathrm{AB}]$ app. A.

It follows from the lemma that $\phi_{i}$ may be written as

$$
\phi_{i}(t)=\left(\tau_{i} \in \mathcal{O}(S)\right) \times \text { element of } \mathbf{C}[t] .
$$

For $n>>0$, we thus have, by definition of the indicial polynomial:

$$
\frac{\tau_{i}}{\left(x-\theta_{i}\right)^{n}} \in \Lambda(\text { something in } \mathcal{O}(X))+\frac{1}{\left(x-\theta_{i}\right)^{n-1}} \mathcal{O}(S)[x] .
$$

A similar statement holds for the section at infinity (instead of $\theta_{i}$ ). Let $U$ denote the complement of the divisor $\left(\tau_{\infty} \cdot \Pi \tau_{i}=0\right)$ in $S$.

Confronting this to the decomposition

$$
\mathcal{O}(X)=\mathcal{O}(S)[x] \oplus \bigoplus_{i} \frac{1}{x-\theta_{i}} \mathcal{O}(S)\left[\frac{1}{x-\theta_{i}}\right]
$$

we arrive at the desired conclusion that the localization of $\mathcal{O}(X) / \Lambda(\mathcal{O}(X))$ at $\left(\tau_{\infty} . \Pi \tau_{i}=0\right)$ is finitely generated over $\mathcal{O}(U)$.

Remark. If $\nabla$ is regular, then the $\tau_{i}$ are units, and $U=S$ in this case. According to the variant of the lemma of dévissage, one can conclude that $R_{d R}^{i} f_{*}$ of a regular connection, with respect to a general smooth morphism $f$, is a vector bundle when restricted to some dense open subset $U \subset S$ which depends only on $f$, cf. [AB]III. 6,8 .

By similar arguments of dévissage, one can prove:

- (generic) base change for $R_{d R}^{i} f_{*}[\mathrm{AB}] \mathrm{III} .5$ : 
Theorem 1.3.4. (Assumptions as in 1.3.1) There is a dense open subset $U \subset S$ with the following property. For any smooth complex variety $S^{\sharp}$ and any morphism $u: S^{\sharp} \longrightarrow S$, let us construct the fibered diagram

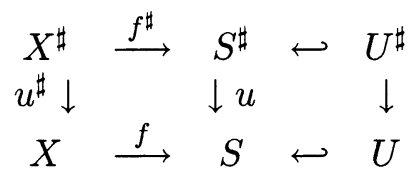

Then for any $i$, the restriction to $U^{\sharp}$ of the base change morphism

$$
u^{*} R_{D R}^{i} f_{*}(\mathcal{M}, \nabla) \longrightarrow R_{D R}^{i} f_{*}^{\sharp} u^{\sharp *}(\mathcal{M}, \nabla)
$$

is an isomorphism.

- stability of regularity under $R_{d R}^{i} f_{*} \quad[\mathrm{AB}] \mathrm{III} .8$ :

Theorem 1.3.5. If $\nabla$ is regular, then for any $i$, the Gauss-Manin connection on $R_{d R}^{i} f_{*}(\mathcal{M}, \nabla)$ is regular.

Here we apply the lemma of dévissage with $\mathcal{P}=$ regularity (cf. [K2] for a "classical" proof which uses resolution of singularities).

- One can also take $\mathcal{P}=$ regularity + having exponents in a given rational vector subspace of $\mathbf{C}$ containing $1 \ldots$

\subsection{Comparison theorem.}

It is crucial for our proof to state the comparison theorem in the relative setting [AB]IV.3:

Theorem 1.4.1. Let $f: X \rightarrow S$ be a smooth morphism, and $(\mathcal{M}, \nabla)$ be a vector bundle with integrable regular connection. Then there is an open dense subset $U \subset S$ (independent of $(\mathcal{M}, \nabla)$ ) such that for any $i$, the canonical morphism

$$
\left(R_{D R}^{i} f_{*}(\mathcal{M}, \nabla)\right)^{a n} \longrightarrow\left(R_{D R}^{i} f_{*}^{a n}\left(\mathcal{M}^{a n}, \nabla^{a n}\right)\right)
$$

induces an isomorphism of analytic vector bundles with connection over $U^{a n}$.

By dévissage along the previous lines, it is possible to reduce the proof to the case when $f$ is a rational elementary fibration (in which case $U=S$ ). Moreover, by base change in De Rham cohomology (cf. 1.3.4), one can further reduce to the case when $S$ is a point (this reduction is not necessary, but simplifies the exposition, cf. [AB]IV.3.4, 3.5). The statement then amounts to the following

Proposition 1.4.2. Let $X=\operatorname{Spec} \mathbf{C}\left[x, \frac{1}{\prod_{i=1}^{r}\left(x-\theta_{2}\right)}\right]$, and let $(E, \nabla)$ be a projective $\mathcal{O}(X)$-module of finite rank with a regular connection. Then $(\mathrm{Co}) \operatorname{Ker}_{E} \nabla\left(\frac{d}{d x}\right) \cong(\mathrm{Co}) \operatorname{Ker}_{E \otimes \mathcal{O}\left(X^{a n}\right)} \nabla\left(\frac{d}{d x}\right)$. 
Let $\mathcal{M}_{\theta_{i}}$ be the field of germs of meromorphic functions at $\theta_{i}$, and let $A_{i}$ denote the (differential) ring generated by $\mathcal{M}_{\theta_{i}}, \log \left(x-\theta_{i}\right)$ and the $\left(x-\theta_{i}\right)^{a}$ for $a \in \mathbf{C}$ (or just for $a$ running through the exponents at $\theta_{i}$ ). For $i=0$, we take the variant at $\infty$.

The proof of 1.4.2. relies on the following classical

Lemma (Frobenius). (Assumptions as in 1.4.2) Due to the regularity of $\nabla, E$ is solvable in $A_{i}$, i.e. $\left(E \otimes A_{i}\right)^{\nabla\left(\frac{d}{d x}\right)} \otimes_{\mathbf{C}} A_{i} \stackrel{\sim}{\rightarrow} E \otimes A_{i}$.

The deduction of 1.4.2 from Frobenius' lemma is formal and uses the following abstract "comparison criteria" in differential algebra.

Let $(C, \partial)$ be a differential Q-algebra, $A, B$ be differential sub-algebras, such that $A^{\partial}=C^{\partial}$ (equality of rings of constants), $A$ is faithfully flat over $A^{\partial}$, and $\partial_{\mid A}$ is surjective onto $A$.

Criterion 1.4.3. Let $E$ be an $(A \cap B)[\partial]$-module, projective of finite rank $\mu$ as a module over $A \cap B$. Assume that $E$ is solvable in $A$, i.e. $(E \otimes$ $A)^{\partial} \otimes_{A^{\partial}} A \stackrel{\sim}{\rightarrow} E \otimes A$.

Then $\operatorname{Ker}_{E} \partial \cong \operatorname{Ker}_{E \otimes B} \partial$ and $\operatorname{Coker}_{E} \partial \hookrightarrow \operatorname{Coker}_{E \otimes B} \partial$ is injective.

Criterion 1.4.4. Assume moreover that $C$ (resp. A) is a product of differential Q-algebras without zero divisors $C_{i}$ (resp. $A_{i} \subset C_{i}$ ), $i=0,1, \ldots, r$ containing a differential subalgebra $C_{i}^{\prime}\left(\right.$ resp. $\left.A_{i}^{\prime}:=A_{i} \cap C_{i}^{\prime}\right)$ such that:

- $\partial: C_{i} \rightarrow C_{i}$ is surjective and $C_{i}$ is faithfully flat over $C_{i}^{\partial}$;

- the composed homomorphism of differential algebras $B \rightarrow C \stackrel{\mathrm{pr}_{i}}{\longrightarrow} C_{i}$ factors through an injection $B \hookrightarrow C_{i}^{\prime}$;

- $C_{i}=T_{i} \oplus \bigoplus_{j \geq 0} C_{i}^{\prime} \partial_{i}^{-j} 1$ for some $C_{i}^{\prime}[\partial]$-submodule $T_{i}$, with $\partial_{i}=u_{i} \partial$ for some unit $u_{i}$ in $A_{i} \cap C_{i}^{\prime}, \partial_{i}\left(\partial_{i}^{-j} 1\right)=\partial_{i}^{-j+1} 1$;

- $C_{0}^{\prime} \subset\left(C_{0}^{\prime} \cap A_{0}\right)+\operatorname{pr}_{0} B$, and, for $i>0, C_{i}^{\prime} \subset\left(C_{i}^{\prime} \cap A_{i}\right)+\bigcap_{j<i} \operatorname{pr}_{i}\left(B \cap A_{j}\right)$.

Then $\operatorname{Coker}_{E} \partial \rightarrow \operatorname{Coker}_{E \otimes B} \partial$ is surjective.

cf. [AB]IV.2.

Application of the criteria: we take

$A_{i}$ as in Frobenius'lemma,

$C_{i}^{\prime}=\bigcap_{\epsilon>0} \mathcal{O}\left(D\left(\theta_{i}, \epsilon\right) \backslash\left\{\theta_{i}\right\}\right)$ (germs of meromorphic functions in small punctured disk around $\theta_{i}$ ),

$$
\begin{aligned}
& C_{i}=A_{i} \cdot C_{i}^{\prime}, \\
& C=\prod C_{i}, A=\prod A_{i}, \partial=\frac{d}{d x}, \\
& B=\mathcal{O}\left(X^{a n}\right) .
\end{aligned}
$$

Then $A \cap B=\mathcal{O}(X)=\mathbf{C}\left[x, \frac{1}{\prod_{i=1}^{r}\left(x-\theta_{i}\right)}\right]$, diagonally embedded into $A$. One has $A_{i}^{\partial}=C_{i}^{\partial}=\mathbf{C}$ and $A_{i} \cap C_{i}^{\prime}=\mathcal{M}_{\theta_{i}}$.

We also set $u_{i}=x-\theta_{i}, \partial_{i}^{-j} 1=\frac{\log ^{j}\left(x-\theta_{i}\right)}{j !}, T_{i}=\sum_{a \notin \mathbf{Z}} C_{i}^{\prime}\left(x-\theta_{i}\right)^{a}$.

The fact that $E$ is solvable in $A$ is Frobenius'lemma (applied at various singularities). 
1.5. Riemann's existence theorem in higher dimension: an elementary approach.

Theorem (Grauert-Remmert). Let $X$ be a smooth complex algebraic variety. Then the natural functor

$\{$ algebraic étale coverings of $X\} \longrightarrow$

\{topological unramified finite coverings of $X(\mathbf{C})$ \}

is an equivalence of categories.

The problem of essential surjectivity of this functor, sometimes called Riemann's existence problem, was settled by Grauert and Remmert [GR] and subsequently by Grothendieck using Hironaka's resolution of singularities.

Using dévissage à la Artin, and special cases of the comparison theorem for De Rham cohomology, it is in fact possible to give an elementary proof of the Grauert-Remmert theorem by reduction to the one-dimensional case.

Recall that a topological unramified covering of $X(\mathbf{C})$ admits a canonical structure of an analytic variety $\mathcal{Y}$, endowed with a holomorphic étale morphism $\pi: \mathcal{Y} \longrightarrow X^{a n}$. In the finite case, we have equivalences of categories

$\left\{\right.$ finite étale coverings $\left.\pi: \mathcal{Y} \longrightarrow X^{a n}\right\} \longrightarrow\left\{\right.$ coherent $\mathcal{O}_{X^{a n} \text {-modules }}$

with integrable connection $(\mathcal{M}, \nabla)$, endowed with a horizontal

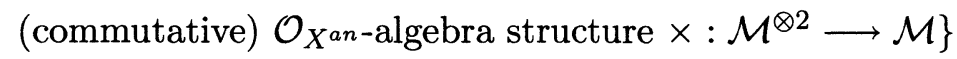

$\longrightarrow$ étale $\mathcal{O}_{X^{a n} \text {-algebras }}(\mathcal{M}, \times)$, coherent as $\mathcal{O}_{X^{a n} \text {-modules }}$

given by

$$
\pi \longmapsto\left(R_{D R}^{0} \pi_{*}\left(\mathcal{O}_{\mathcal{Y}}, d\right), \times\right)=\left(\pi_{*} \mathcal{O}_{\mathcal{Y}}, \nabla, \times\right) \longmapsto\left(\pi_{*} \mathcal{O}_{\mathcal{Y}}, \times\right),
$$

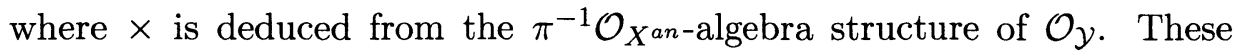
equivalences also take place in the algebraic categories:

\{finite étale coverings $\pi: Y \longrightarrow X\} \longrightarrow\left\{\right.$ coherent $\mathcal{O}_{X}$-modules

with integrable regular connection $(\mathcal{M}, \nabla)$, endowed with a horizontal

$$
\begin{gathered}
\text { (commutative) } \left.\mathcal{O}_{X} \text {-algebra structure } \times: \mathcal{M}^{\otimes 2} \longrightarrow \mathcal{M}\right\} \\
\longrightarrow\left\{\text { finite étale } \mathcal{O}_{X^{-}} \text {-algebras } \mathcal{M}\right\}
\end{gathered}
$$

We refer to $[\mathrm{AB}]$ app. $\mathrm{E}$ for a proof of the Grauert-Remmert theorem along these lines (which does not use resolution of singularities, nor any "hard result" in complex analysis). 


\subsection{Translation into the $p$-adic setting: the Kiehl-Baldassarri comparison theorem.}

The proof of the Grothendieck-Deligne comparison theorem which we have sketched is so formal that it translates almost literally into the $p$-adic setting. In this setting, $X$ is a smooth algebraic variety over $\mathbf{C}_{p}$, and $X^{a n}$ denotes the associated rigid-analytic variety.

With the same notation and assumptions as in 1.4.2 (but interpreted in the $p$-adic context), Frobenius'lemma has the following $p$-adic counterpart:

Lemma (Clark). Assume that the abelian group generated by 1 and the exponents of $\nabla$ does not contain any Liouville number. Then, due to the regularity of $\nabla, E$ is solvable in $A_{i}$, i.e. $\left(E \otimes A_{i}\right)^{\nabla\left(\frac{d}{d x}\right)} \otimes_{\mathbf{C}} A_{i} \stackrel{\sim}{\rightarrow} E \otimes A_{i}$.

The diophantine condition on the exponents is specific to the $p$-adic case and unavoidable: for instance the series

$$
\sum \frac{x^{n}}{a+n}=x^{-a} \int \frac{x^{a} d x}{1-x}
$$

(which satisfies a first order inhomogeneous linear differential equation) may diverge $p$-adically if $a$ is a Liouville number, i.e. very-well approximated by integers.

Building on Clark's lemma, the same arguments as in $\S 1.4$ apply and give a proof of the following generalization of Baldassarri's theorem in the relative setting:

Theorem 1.6.1. Let $f: X \rightarrow S$ be a smooth morphism, and $(\mathcal{M}, \nabla)$ be a vector bundle with integrable regular connection. We assume that the additive subgroup of $\mathbf{C}_{p}$ generated by 1 and the exponents of $\nabla$ contains no Liouville number. Then there is an open dense subset $U \subset S$ (independent of $(\mathcal{M}, \nabla))$ such that for any $i$, the canonical morphism

$$
\left(R_{D R}^{i} f_{*}(\mathcal{M}, \nabla)\right)^{a n} \longrightarrow\left(R_{D R}^{i} f_{*}^{a n}\left(\mathcal{M}^{a n}, \nabla^{a n}\right)\right)
$$

induces an isomorphism of analytic vector bundles with connection over $U^{a n}$.

cf. [AB] IV 4.1.

One recovers Kiehl's theorem as the following special case:

Corollary 1.6.2. $H_{d R}^{*}(X) \cong H_{d R}^{*}\left(X^{a n}\right)$.

2. Comparison theorem between algebraic and analytic De Rham cohomology with arbitrary $p$-adic coefficients

\subsection{Introduction.}

The Frobenius and Clark lemmata are not quite faithful reflections of each other in the complex and $p$-adic worlds respectively. We have already noticed the diophantine condition in Clark's lemma. On the other hand, 
while the convergence property asserted by Frobenius'lemma is specific to regular connections, the convergence property asserted by Clark's lemma extend to irregular connections in the following way.

Let $K$ be an algebraically closed field of characteristic 0 , complete under a non-archimedean absolute value $\mid$ (e.g. $K=\mathbf{C}_{p}$ ). Let $K_{0}$ be an algebraically closed subfield of $K$ which does not contain Liouville numbers, i.e. for every $a \in K_{0}$, the series $\sum_{n \in \mathbf{N}, n \neq-a} \frac{x^{n}}{a+n}$ converges (e.g. $K_{0}=\overline{\mathbf{Q}}$ ).

Let us consider a monic differential operator $\Lambda \in K_{0}\left[x, \frac{1}{\prod_{i=1}^{r}\left(x-\theta_{i}\right)}\right]\left[\frac{d}{d x}\right]$, with $\theta_{i} \in K_{0}$. Let $\mathcal{M}_{\theta_{i}}$ be the field of germs of meromorphic functions at $\theta_{i}$, and let now $A_{i}$ denote the (differential) ring generated by $\mathcal{M}_{\theta_{i}}, \log \left(x-\theta_{i}\right)$, the $\left(x-\theta_{i}\right)^{a}$ and $\exp \left(b\left(x-\theta_{i}\right)^{r}\right)$ for $a, b \in K_{0}, r \in \mathbf{Q}_{<0}$. For $i=0$, we take the variant at $\infty$.

Lemma (Clark-Baldassarri). $\Lambda$ is solvable in $A_{i}$.

cf. [B1]. The lemma asserts in particular the convergence of any formal power series which is a solution of $\Lambda$ (in strong contrast with the complex case).

This and similar peculiar features of non-archimedean differential equations led Baldassarri to conjecture in 1987 that the comparison theorem between algebraic and analytic De Rham cohomology might hold for arbitrary, not necessary regular, coefficients [B2]. This conjecture has now been proved [AB]IV.6.1:

Main theorem. Let $X$ be a smooth algebraic $K_{0}$-variety, and let $(\mathcal{M}, \nabla)$ be a coherent $\mathcal{O}_{X}$-module with integrable connection. Then the natural map

$$
H_{d R}^{*}\left(X_{K},\left(\mathcal{M}_{K}, \nabla_{K}\right)\right) \rightarrow H_{d R}^{*}\left(X_{K}^{a n},\left(\mathcal{M}_{K}^{a n}, \nabla_{K}^{a n}\right)\right)
$$

is an isomorphism.

In degree 0 , this gives:

Corollary. The functor of analytification

$$
\left(\mathcal{M}_{K}, \nabla_{K}\right) \mapsto\left(\mathcal{M}_{K}^{a n}, \nabla_{K}^{a n}\right)
$$

of modules with integrable connection defined over $K_{0}$ is fully faithful.

However, it is not essentially surjective. This is again in strong contrast with the complex case, where the functor of analytification is essentially surjective but not fully faithful (unless one restricts to regular connections).

Apart from the case of regular coefficients, a special case of the main theorem had been previously proved by two different methods [B1][C], namely the case when $X$ is a curve. 


\subsection{Strategy of proof; key lemma.}

2.2.1. The overall strategy is the same as for theorem 1.6 .1 (case of regular coefficients):

a) dévissage using towers of elementary fibrations,

b) study of the case of (rational) elementary fibrations.

Its core is the following

Key lemma. There is a finite open affine cover $\left(U_{\alpha}\right)$ of $X$ and, for each $\alpha$, an elementary fibration $f_{\alpha}: U_{\alpha} \rightarrow S_{\alpha}$

$$
\begin{array}{ccccc}
U_{\alpha} & \hookrightarrow & \bar{U}_{\alpha} & \hookleftarrow & Z_{\alpha}=\bar{U}_{\alpha} \backslash U_{\alpha} \\
& f_{f_{\alpha}} & & g_{\alpha} & \\
& & \downarrow & \swarrow &
\end{array}
$$

such that for $j=0,1$ :

(i) $R_{D R}^{j} f_{\alpha *}\left((\mathcal{M}, \nabla)_{\mid U_{\alpha}}\right)$ is a vector bundle on $S_{\alpha}$,

(ii) $\left(R_{D R}^{j} f_{\alpha *}\left((\mathcal{M}, \nabla)_{\mid U_{\alpha}}\right)\right)^{a n} \cong\left(R_{D R}^{j} f_{\alpha *}^{a n}\left(\left(\mathcal{M}^{a n}, \nabla^{a n}\right)_{\mid U_{\alpha}^{a n}}\right)\right)$.

2.2.2. Given point $i$ ) of the key lemma, point $i i$ ) is just a variant of theorem 1.6.1 with irregular coefficients, in the case of an elementary fibration. In is settled in the same way, reducing to the case of a rational elementary fibration and using the Clark-Baldassarri lemma instead of Clark's lemma, cf. [AB]IV.5.2.

2.2.3. Let us now deduce the main theorem from the key lemma.

We consider a cover $\left(U_{\alpha}\right)$ as in the key lemma. For $\underline{\alpha}=\left(\alpha_{0}, \ldots, \alpha_{p}\right)$, $\alpha_{0}<\cdots<\alpha_{p}$, we set $U_{\underline{\alpha}}=U_{\alpha_{0}} \cap \cdots \cap U_{\alpha_{p}}$. There is a natural morphism of Čech spectral sequences,

$$
\begin{array}{ccc}
\oplus_{\alpha_{0}<\cdots<\alpha_{p}} H_{D R}^{i-p}\left(U_{\underline{\alpha}},(\mathcal{M}, \nabla)\right) \otimes K & \Rightarrow & H_{D R}^{i}(X,(\mathcal{M}, \nabla)) \otimes K \\
\downarrow \varphi_{i-p} & \downarrow \varphi_{i} \\
\oplus_{\alpha_{0}<\cdots<\alpha_{p}} H_{D R}^{i-p}\left(U_{\underline{\alpha} K}^{a n},\left(\mathcal{M}^{a n}, \nabla^{a n}\right)\right) & \Rightarrow & H_{D R}^{i}\left(X_{K}^{a n},\left(\mathcal{M}^{a n}, \nabla^{a n}\right)\right) .
\end{array}
$$

By induction on $i$, we are left with proving the theorem for $X=U_{\alpha}$.

On the other hand, there is a natural morphism of Leray spectral sequences

$$
\begin{array}{ccc}
\oplus_{0 \leq j \leq i} H_{D R}^{i-j}\left(S_{\alpha}, R_{D R}^{j} f_{\alpha *}\left((\mathcal{M}, \nabla)_{\mid U_{\alpha}}\right)\right) \otimes K & \Rightarrow & H_{D R}^{i}(X,(\mathcal{M}, \nabla)) \otimes K \\
\downarrow \varphi_{i-j}^{\prime} & \downarrow \\
\oplus_{0 \leq j \leq i} H_{D R}^{i-j}\left(S_{\alpha K}^{a n},\left(R_{D R}^{j} f_{\alpha *}\left((\mathcal{M}, \nabla)_{\mid U_{\alpha}}\right)^{a n}\right)\right. & \downarrow \varphi_{j}^{\prime \prime} \\
\oplus_{0 \leq j \leq i} H_{D R}^{i-j}\left(S_{\alpha K}^{a n}, R_{D R}^{j} f_{\alpha *}^{a n}\left(\left(\mathcal{M}^{a n}, \nabla^{a n}\right)_{\mid U_{\alpha}^{a n}}\right)\right. & \Rightarrow & H_{D R}^{i}\left(X_{K}^{a n},\left(\mathcal{M}^{a n}, \nabla^{a n}\right)\right) .
\end{array}
$$


By point $i i$ ) of the key lemma, we know that $\varphi_{j}^{\prime \prime}$ is an isomorphism. By point $i$ ) of the key lemma and induction on the dimension, we may assume that $\varphi_{i-j}^{\prime}$ is an isomorphism. Hence the right vertical arrow $\varphi_{i}$ is an isomorphism.

2.2.4. The main difficulty in the key lemma is to find elementary fibrations $f_{\alpha}: U_{\alpha} \rightarrow S_{\alpha}$ (the $U_{\alpha}$ covering all of $X$ ), such that $R_{D R}^{1} f_{\alpha *}\left((\mathcal{M}, \nabla)_{\mid U_{\alpha}}\right)$ is $\mathcal{O}_{S_{\alpha}}$-coherent. This difficulty will lead us to a detailed study of the ramification properties of irregular connections.

In order to understand the (purely algebro-geometric) nature of the problem, let us recall our approach to the coherence of $R_{D R}^{1} f_{*}$ in 1.3.2. We again consider the case of a rational elementary fibration with affine base:

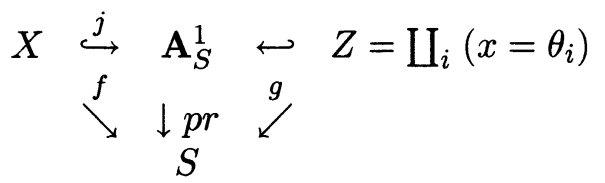

with $\theta_{i}-\theta_{j} \in \mathcal{O}(S)^{*}, x$ being the "vertical" coordinate on $\mathbf{A}^{1}$.

We were assuming that the connection $\nabla$ was cyclic with respect to the $x$-coordinate, i.e. given by a differential operator

$$
\Lambda=\sum_{0}^{\mu} \gamma_{k} \frac{d^{k}}{d x^{k}} \in \mathcal{O}(X)\left[\frac{d}{d x}\right]
$$

so that $R_{d R}^{1} f_{*}(\mathcal{M}, \nabla)=\operatorname{Coker} \Lambda$.

The indicial polynomial $\phi_{i}$ of $\Lambda$ at $\theta_{i}$ was written as

$$
\phi_{i}(t)=\left(\tau_{i} \in \mathcal{O}(S)\right) \times \text { element of } \mathbf{C}[t] .
$$

Combining the decomposition

$$
\mathcal{O}(X)=\mathcal{O}(S)[x] \oplus \bigoplus_{i} \frac{1}{x-\theta_{i}} \mathcal{O}(S)\left[\frac{1}{x-\theta_{i}}\right]
$$

with the process of reduction of denominators

$$
\frac{\tau_{i}}{\left(x-\theta_{i}\right)^{n}} \in \Lambda(\text { element of } \mathcal{O}(X))+\frac{1}{\left(x-\theta_{i}\right)^{n-1}} \mathcal{O}(S)[x] \text {, for } n>>0,
$$

(and similarly at infinity), we arrived at the conclusion that $\operatorname{Coker} \Lambda$ is finitely generated after localization on the complement of the divisor $\left(\tau_{\infty} \cdot \prod \tau_{i}=0\right)$ in $S$.

If we try to copy this approach in the context of the key lemma, we are faced with two questions:

Q.1. The existence of cyclic vector in the neighborhood of point which is not a singularity is well-known [K3], but does there exist a cyclic vector in the neighborhood of singularities?

Q.2. Assuming that we can reduce to the cyclic situation, can we achieve 
moreover that the leading coefficients $\tau_{i}$ of the indicial polynomials are units in $\mathcal{O}\left(S_{\alpha}\right)$ ?

2.2.5. It turns out that the first question has a negative answer in general: indeed, let $X$ be the $(x, y)$-plane, $Z=$ the $y$-axis, $\mathcal{M}=\mathcal{O}_{X \backslash Z} e_{1} \oplus \mathcal{O}_{X \backslash Z} e_{2}$, where the first (resp. second) factor is endowed with the trivial connection (resp. the connection with formal solution $e^{-\frac{y}{x}}$ ). If $v=a e_{1}+b e_{2}, \frac{\partial}{\partial x} v=$ $\frac{\partial a}{\partial x} e_{1}+\left(\frac{\partial b}{\partial x}-\frac{b y}{x^{2}}\right) e_{2}$; by looking at the term of lowest $x$-order, we see that for any affine open neighborhood $U$ of the origin, the determinant $a \frac{\partial b}{\partial x}-b \frac{\partial a}{\partial x}-$ $\frac{a b y}{x^{2}}$ cannot be a unit in $\mathcal{O}(U \backslash(Z \cap U))$, hence there is no cyclic vector for $\nabla\left(\frac{\partial}{\partial x}\right)$ in $\mathcal{O}(U \backslash(Z \cap U))$.

We are going to give criteria for the above questions in terms of Newton polygons. The next three sections are purely algebraic in nature and devoted to the formal study of irregular connections. In these sections, $K$ is any algebraically closed field of characteristic 0 .

\subsection{Newton polygons.}

2.3.1. Let us consider a differential operator $\Lambda=\sum \gamma_{i j} \frac{x^{j} d^{i}}{d x^{2}}$, monic, of degree $\mu$ in $\frac{d}{d x}\left(\gamma_{i j} \in K\right)$.

We recall that the Newton polygon $N P_{0}(\Lambda)$ of $\Lambda$ at $x=0$ is the convex hull of the quadrants $\left\{u \leq i, v \geq j-i+\mu \mid \gamma_{i j} \neq 0\right\} \subset \mathbf{R}^{2}$. The vertices of $N P_{0}(\Lambda)$ are among the points $\left(i, \operatorname{ord}_{x}\left(\gamma_{i}\right)-i+\mu\right), 0 \leq i \leq \mu$. The vertical edge of $N P_{0}(\Lambda)$ is $\{u=\mu, v \geq 0\}$.

If we write $x^{\mu} \Lambda=\left(\frac{x}{d x}\right)^{\mu}-\sum_{i=0}^{\mu-1} \beta_{i}(x)\left(\frac{x}{d x}\right)^{i}, \beta_{\mu}(x)=1$, then $N P_{0}(\Lambda)$ can also be described as the convex hull of the quadrants $\{u \leq i, v \geq$ $\left.\operatorname{ord}_{x}\left(\beta_{i}\right)\right\}, 0 \leq i \leq \mu$.

The height of $N P_{0}(\Lambda)$ (i.e. the maximal distance between the ordinates of the vertices) is called the irregularity of $\Lambda$. All finite slopes of $N P_{0}(\Lambda)$ are $\geq 0$ and the maximal one is $\max \left(0, \max _{j=0, \ldots, \mu-1}\left(-\frac{v\left(\gamma_{j}\right)}{\mu-j}-1\right)\right)$. The Newton polygon is a quadrant if and only if 0 is a regular singularity.

It turns out that the Newton polygon is an invariant of the differential module $K((x))\left[\frac{d}{d x}\right] / \Lambda K((x))\left[\frac{d}{d x}\right]$ (it does not depend on the particular choice of a cyclic vector). This remark allows to define the Newton polygon of any differential module over $K((x))$ at 0 (resp. over $K(x)$ at any point, including $\infty$ ).

2.3.2. Let us consider an elementary fibration (cf. 1.2.2)

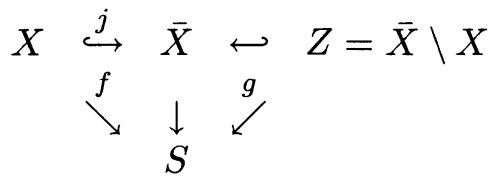


and let $\mathcal{M}$ be a vector bundle on $X$ endowed with a relative connection

$$
\nabla_{r e l}: \mathcal{M} \rightarrow \Omega_{X / S}^{1} \otimes \mathcal{M}
$$

For each point $Q$ of $Z$, we can form the Newton polygon at $Q$ of the fiber of $\left(\mathcal{M}, \nabla_{\text {rel }}\right)$ above $g(Q)$. This gives rise to a polygonal function $N_{f}=N_{f}\left(\nabla_{r e l},.\right)$ on $Z$.

Proposition 2.3.3. $N_{f}$ is lower semi-continuous, i.e. for any given polygon $N,\left\{Q \in Z \mid N_{f}(Q) \subset N\right\}$ is closed in $Z$ (or else: $N_{f}$ is constructible, and for any specialization $Q \longrightarrow P$ in $\left.Z, N_{f}(P) \subseteq N_{f}(Q)\right)$.

cf. [AB]II.4.2.3.

We now assume that $\nabla_{\text {rel }}$ comes from an absolute integrable connection $\nabla$ on $X$. We also assume that $Z$ is given by a disjoint union smooth irreductible divisors $Z_{i}$. Let $\partial$ be a vertical derivation (e.g. $\frac{\partial}{\partial x}$ for some local vertical coordinate $x$ ). For question Q.1, we have the following

Criterion 2.3.4. Assume that:

i) $N_{f}\left(\nabla_{(\text {internal }) \text { End }},.\right)$ is constant on $Z$,

ii) the Turrittin index (cf. next section) of $\nabla$ at each $Z_{i}$ is 1 (this is slighly stronger than requiring integrality of the slopes),

iii) $\mathcal{M}$ extends to a vector bundle on $\bar{X}$.

Then there exists a flat morphism $h: S^{\prime} \rightarrow S$, finite over its image, with $\operatorname{codim}\left(S \backslash h\left(S^{\prime}\right)\right) \geq 2$ and such that every $Q \in S^{\prime}$ has an open neighborhood $U(Q)$ in $\bar{X} \times_{S} S^{\prime}$ such that $\nabla_{U(Q) \cap X \times{ }_{S} S^{\prime}}$ is cyclic with respect to $\nabla(\partial)$.

For question Q.2, we have the following

Criterion 2.3.5. Assume that $N_{f}(\nabla,$.$) is constant on Z$. Then for any cyclic vector of $\nabla(\partial)$ (if any), with associated differential operator $\Lambda$, the leading coefficient of the indicial polynomial of $\Lambda$ at $Z$ is a unit in $\mathcal{O}(Z)$.

cf. [AB]II.7.2.9 and 7.1.2. The proof of both criteria depends on a certain relative version of the Turrittin-Levelt-Hukuhara decomposition theorem of irregular formal connections.

\subsection{Formal decomposition of an integrable connection along a singular divisor.}

2.4.1. Let $Y$ be a smooth affine $K$-variety, and let $Z \subset Y$ be smooth divisor. We choose local coordinates $t=x_{1}, \ldots x_{d}$ such that $t=0$ is a local equation for $Z$.

There is a canonical decomposition of the formal completion $\hat{Y}_{Z}$ :

$$
\hat{Y}_{Z} \cong \hat{\mathbf{A}}^{1} \times Z, \mathcal{O}\left(\hat{Y}_{Z}\right) \cong \mathcal{O}(Z)[[t]] \text {. }
$$

cf. [AB]I.1.4.5. In other words, the vector field $\frac{\partial}{\partial t}$ admits a canonical system of formal integral curves $\hat{C}_{Q} \cong \hat{\mathbf{A}}^{1}, Q \in Z$. 
Let $\mathcal{E}$ be a projective $\mathcal{O}(Z)((t))$-module of rank $\mu$ endowed with an integrable connection $\left(\frac{\partial}{\partial t}=\frac{\partial}{\partial x_{1}}, \ldots, \frac{\partial}{\partial x_{d}}\right.$ act on $\left.\mathcal{E}\right)$.

Forgetting about the variables $x_{2}, \ldots, x_{d}$, the Turrittin-Levelt-Hukuhara theorem tells us that there exists: • a finite extension of $K(Z)((t))$ with ramification index $e \mid \mu$ ! (the so-called Turrittin index at $t=0$ ) [we may and shall write this extension in the form $K\left(Z^{\prime}\right)\left(\left(t^{\prime}\right)\right)$, with $t^{\prime}=t^{1 / e}, Z^{\prime}$ normal and finite over $Z$ ],

- polynomials $P_{1}, \ldots, P_{r} \in K\left(Z^{\prime}\right)\left[\frac{1}{t^{\prime}}\right]$, well-defined modulo $\mathbf{Z}$, such that the following decomposition of $\frac{\partial}{\partial t^{\prime}}$-modules holds:

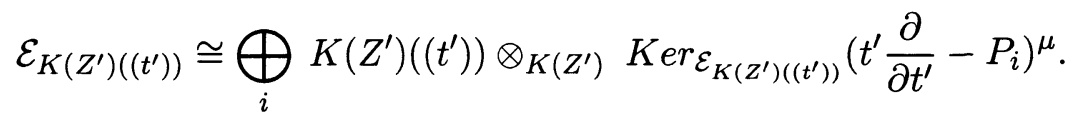

cf. [AB]II.3.1. The following result is an "integral" refinement of this decomposition $\left(K\left(Z^{\prime}\right)\right.$ replaced by $\left.\mathcal{O}\left(Z^{\prime}\right)\right)$.

Theorem 2.4.2. Assume that the horizontal side of the Newton polygon of the internal End of $\nabla$ restricted to the integral curve $\hat{C}_{Q}$ does not depend on $Q \in Z$. Then:

i) the polynomials $P_{i}$ belong to $\mathcal{O}\left(Z^{\prime}\right)\left[\frac{1}{t^{\prime}}\right]$,

ii) the Turrittin-Levelt-Hukuhara decomposition holds in integral form:

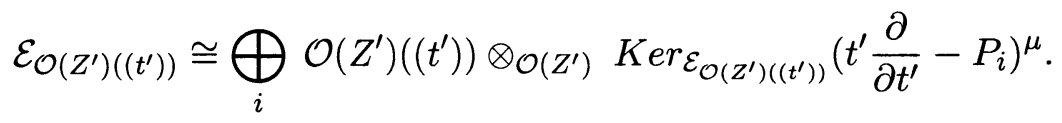

The proof is long and delicate ([AB]II.6), in the spirit of $x_{i}$-adic analysis. It is likely that the technical assumption of 2.4.2 is not necessary (cf. [AB]II.6.13 for a discussion). Let us also note that there is a $p$-adic refinement of 2.4.2, which can be viewed as a higher dimensional generalization of the Clark-Baldassarri lemma [AB]IV.7.

It follows from 2.4.2 that the slope filtration of $\mathcal{E}$ is horizontal with respect to $\frac{\partial}{\partial t}=\frac{\partial}{\partial x_{1}}, \ldots, \frac{\partial}{\partial x_{d}}$.

\subsection{The NP-stratification of a singular divisor.}

2.5.1. The main assumption in criteria 2.3 .4 and 2.3.5. concerns the (non-) variation of the Newton polygon in the fibers of an elementary fibration. The semi-continuity property 2.3.3. is not enough for our purpose, and we sketch here a more precise study.

Let $Y, Z, t=x_{1}, \ldots x_{d}$ be as in 2.4.1. Instead of a formal module with connection, we now consider an algebraic vector bundle $\mathcal{M}$ on $Y \backslash Z$ with integrable connection $\nabla$. Working with an algebraic closure $K(Z)$ instead of $K$, the method of 2.3.1. allows to define the Newton polygon $N P_{Z}(\nabla)$ at (the generic point of) $Z$. We wish to compare this Newton polygon with the Newton polygon $N P_{Q}\left(\nabla_{\mid C \backslash Q}\right)$ at various point $Q \in Z$ of the restriction of $\nabla$ to a curve $C$ in $Y$ meeting $Z$ transversally at $Q$. 
Theorem 2.5.2. There is an open dense subset $Z_{0} \subset Z$ such that for any locally closed curve $C$ which meets $Z$ transversally at some point $Q \in$ $Z_{0}, N P_{Q}\left(\nabla_{\mid C \backslash Q}\right)=N P_{Z}(\nabla)$.

(Again, the proof has some flavor of ultrametric analysis [AB]II.5.3.1.). By iteration, blowing-up, and using 2.3.3, one obtains the following refinement [AB]II.5.4.:

Theorem 2.5.3 (semi-continuity). There is a finite set $\mathcal{N}$ of polygons $\subset \mathbf{R}^{2}$ with integral vertices, and a constructible partition $Z=\amalg_{N \in \mathcal{N}} Z_{N}$ such that for every $Q \in Z_{N}$ and almost every locally closed curve $C$ which meets $Z$ transversally at $Q$, one has $N P_{Q}\left(\nabla_{\mid C \backslash Q}\right)=N$. Moreover, $\bar{Z}_{N}=$ $\bigcup_{N^{\prime} \subset N} Z_{N^{\prime}}$.

("almost every" is understood in the sense of Zariski topology on the projective bundle of tangent directions). The relationship with stratifications introduced by $\mathrm{Y}$. Laurent and Z. Mebkhout [LMe] would deserve examination.

As a simple illustration of 2.5.3, let us take for $Y$ the affine plane, $Z=$ the $x_{2}$-axis, and let us consider the integrable connection $\nabla$ of rank one with formal solution $\exp \left(x_{2} /\left(x_{1}\right)^{2}\right)$. The stratification is $Z=(Z \backslash 0) \amalg\{0\}$; the stratum $Z \backslash 0$ corresponds to slope 2, the stratum $\{0\}$ corresponds to slope 1 (the excluded curves are those tangent to the $x_{1}$-axis).

\subsection{Indications on the proof of the key lemma.}

We now have all the ingredients to finish the sketch of proof of the main theorem. From the comments in 2.2, it only remains to discuss point $i$ ) of the key lemma. More precisely, given any point $\xi \in X$, we have to find an affine open neighborhood $U=U(\xi)$ of $\xi$ and an elementary fibration $f: U \rightarrow S$ such that $R_{D R}^{1} f_{*}\left((\mathcal{M}, \nabla)_{\mid U}\right)$ is $\mathcal{O}_{S}$-coherent. We refer to [AB]IV.6.6. for complete details.

Let us recall the principle of Artin's construction of elementary fibrations. We may assume that $X$ is affine, of dimension $d$, and we choose a projective completion $\bar{X}$ of $X$. We embed $\bar{X}$ into some $\mathbf{P}^{N}$ using the tensor square of any very ample line bundle. Let $L \subset \mathbf{P}^{N}$ be a sufficiently general linear space of codimension $d-1$ passing through $\xi$, and let $H \subset \mathbf{P}^{N}$ be a sufficiently general hyperplane. The linear projection $\mathbf{P}^{N} \ldots \rightarrow \mathbf{P}^{d-1}$ will then induce, by restriction to a suitable neighborhood $U$ of $\xi$ in $X$, an elementary fibration.

An important point is that for any sufficiently general $L \subset \mathbf{P}^{N}$ will avoid any fixed $T \subset D=\bar{X} \backslash X$ of codimension 1 given in advance. We may and shall fix $T$ in such a way that:

- $D \backslash T$ is a union of smooth components $D_{i}$,

- $\mathcal{M}$ extends to a vector bundle over $\bar{X} \backslash T$, 
- (using 2.5.2) for each $i$, and for any locally closed curve $C$ which meets $D_{i}$ transversally at some point $Q$, the polygonal functions of $Q \in D_{i}$ given by $N P_{Q}\left(\nabla_{\mid C \backslash Q}\right)$ and $N P_{Q}\left(\left(\nabla_{\text {internalEnd }}\right)_{\mid C \backslash Q}\right)$ are constant on $D_{i}$.

After technical reductions to rational elementary fibrations, passing to Kummer coverings etc..., we are left at last with a situation where criteria 2.3.4 and 2.3.5 apply (in particular, the assumptions of non-variation of the Newton polygons are satisfied). We thus have cyclic vectors at disposal, and the leading coefficients of the associated indicial polynomials are units. As was explained in 2.2.4, this provides the coherence of $R_{D R}^{1} f_{*}\left((\mathcal{M}, \nabla)_{\mid U}\right)$ asserted in the key lemma.

\section{References}

[AB] Y. ANDRÉ, F. BALDASSARRI, De Rham cohomology of differential modules on algebraic varieties. Progress in Mathematics 189, Birkäuser (2001).

[Ar] M. ARTin, Comparaison avec la cohomologie classique. Cas d'un schéma lisse. In: M. Artin, A. Grothendieck, J.L. Verdier, Théorie des Topos et Cohomologie Étale des Schémas. Tome 3. Lecture Notes in Math. 305, Springer-Verlag (1973).

[B1] F. BALDASSARRI, Differential modules and singular points of $p$-adic differential equations. Advances in Math. 44 (1982), 155-179.

[B2] F. BALDASSARRI, Comparaison entre la cohomologie algébrique et la cohomologie padique rigide à coefficients dans un module différentiel I. Invent. Math. 87 (1987), 83-99.

[B3] F. BALDASSARRI, Comparaison entre la cohomologie algébrique et la cohomologie padique rigide à coefficients dans un module différentiel II. Math. Ann. 280 (1988), 417-439.

[Bn] J. BERnSTEIN, Lectures on $\mathcal{D}$-modules. Mimeographed notes.

[Bo] A. Borel, Algebraic D-modules. Perspectives in Mathematics, Vol.2, Academic Press (1987).

[C] B. Chiarellotto, Sur le théorème de comparaison entre cohomologies de De Rham algébrique et p-adique rigide. Ann. Inst. Fourier 38 (1988), 1-15.

[Cl] D. CLARK, A note on the p-adic convergence of solutions of linear differential equations. Proc. Amer. Math. Soc. 17 (1966), 262-269.

[D] P. Deligne, Equations Différentielles à Points Singuliers Réguliers. Lecture Notes in Math. 163, Springer-Verlag (1970).

[DMSS] A. DimCA, F. MAAREF, C. SABbah, M. SAito, Dwork cohomology and algebraic $\mathcal{D}$ modules. Math. Ann. 318 (2000), 107-125.

[GR] H. Grauert, R. Remmert, Komplexe Räume. Math. Ann. 136 (1958), 245-318.

[G] A. Grothendieck, On the De Rham cohomology of algebraic varieties. Publications Mathématiques IHES 29 (1966), 93-103.

[K1] N. KATZ, Nilpotent connections and the monodromy theorem. Applications of a result of Turrittin. Publ. Math. IHES 39 (1970), 175-232.

[K2] N. KATZ, The regularity theorem in algebraic geometry. Actes du Congrès Intern. Math. 1970, T.1, 437-443.

[K3] N. KATZ, A simple algorithm for cyclic vectors. Amer. J. of Math. 109 (1987), 65-70.

[KO] N. KATZ, T. ODA, On the differentiation of De Rham cohomology classes with respect to parameters. J. Math. Kyoto Univ. 8, 2 (1968), 199-213.

[Ki] R. KIEHL, Die De Rham Kohomologie algebraischer Mannigfaltigkeiten über einem bewerteten Körper. Publ. Math. IHES 33 (1967), 5-20.

[LMe] Y. Laurent, Z. Mebrhout, Pentes algébriques et pentes analytiques d'un $\mathcal{D}$-module. Ann. Scient. Ecole Normale Sup. (4) 32 (1999), 39-69.

[M] YU. Manin, Moduli fuchsiani. Annali Scuola Normale Sup. Pisa III 19 (1965), 113-126. 
[Me1] Z. Meвкhout, Le théorème de positivité de l'irrégularité pour les $\mathcal{D}_{X}$-modules. In Grothendieck Festschrift vol. III, 83-132, Progress in Mathematics, Birkhäuser (1990).

[Me2] Z. Mевкноuт, Le théorème de comparaison entre cohomologies de De Rham d'une variété algébrique complexe et le théorème d'existence de Riemann. Publ. Math. IHES 69 (1989), 47-89.

Yves ANDRÉ

Institut de Mathématiques

175 rue du Chevaleret

F-75013 Paris

E-mail : andre@math.jussieu.fr 